\title{
Réplique au commentaire du Dr Christian Hess
}

\section{Pierre-François Cuénoud}

Dr, Vice-président de la FMH, Responsable du domaine Tarifs et économie de la santé pour les médecins hospitaliers

Cher Collègue,

C'est avec plaisir que je redécouvre l'indignation de ceux qui, jusqu'en 2011, ont tenté d'imposer un moratoire à l'introduction généralisée des forfaits par cas en Suisse. Votre analyse mérite naturellement quelques commentaires et rectifications.

Vous évoquez tout d'abord la transparence: elle a justement été améliorée par la création d'une structure tarifaire uniforme dans tout le pays, révélant ainsi de manière évidente les différences entre cantons et établissements, mettant le doigt sur les mécanismes de distorsion.

Concernant les coûts, il n'a jamais été question de les diminuer, mais bien plutôt d'enrayer leur progression. La baisse généralisée des taux de base, mettant le couteau sous la gorge des hôpitaux, est typique de cette évolution. Depuis 2012, l'augmentation aussi bien du nombre de cas que des coûts dans le domaine stationnaire est faible, les mécanismes de monitoring étant efficaces: une suractivité manifeste n'est en effet plus rétribuée par les assureurs. Il n'est ainsi possible de développer des prestations inutiles qu'au détriment d'autres qu'il faut alors abandonner. Il est vrai qu'une minorité d'établissements pratiquent une telle stratégie, laissant les cas difficiles et peu rémunérateurs aux grand hôpitaux publics.

Le chapitre de la qualité, bien que prévu dans la loi depuis une vingtaine d'années, n’a été ouvert que récemment. Concomitamment à l'introduction du nouveau financement hospitalier, des indicateurs ont été développés et imposés. Aux yeux de la FMH et comme vous le mentionnez, ils sont encore notoirement insuffisants, l'établissement d'un vrai contrôle de la qualité des indications étant nécessaire conformément à nos revendications relayées par l'Académie Suisse pour la Qualité en Médecine (ASQM).

Correspondance: FMH Dr Pierre-François Cuénoud Elfenstrasse 18 CH-30oo Berne 15 Tél. 0313591111 Fax 0313501112 tion pendant des années et que l'aiguillon d'une nécessaire rationalisation peut être profitable non seulement aux finances, mais également aux processus et à la qualité des traitements.

De manière générale, il est bon que le corps médical accompagne l'évolution des paramètres financiers de la santé de manière critique. Face au constat que de nombreux collègues délaissent la clinique pour des occupations plus administratives, il faut se demander si notre façon de fonctionner dans les hôpitaux a perdu de son attractivité et en rechercher les causes. Si le nombre de postes dédiés à des activités sans contact direct avec les patients a augmenté dans les établissements hospitaliers ces dernières années, leur pourcentage a cependant faibli par rapport aux autres domaines: les postes médicaux sont ceux qui se sont multipliés le plus significativement, devant les soignants. Certaines professions non cliniques sont aussi devenues incontournables à l'image des informaticiens, dont les compétences sont requises lors des nombreuses pannes de nos dossiers patients électroniques.

Globalement, il demeure indispensable, aujourd'hui encore, de distinguer le développement et l'entretien de la structure tarifaire SwissDRG des modifications considérables du financement hospitalier qui ont été introduites simultanément. Stricto sensu, le budget d'exploitation de SwissDRG SA est de 2,3 millions CHF par an, bien loin des centaines de millions que vous mentionnez.

Objectivement, pour qui sait faire la part des choses sans naïveté idéaliste, la pratique généralisée des forfaits par cas dans notre pays n'a pas eu d'impact révélateur mesurable jusqu'à présent et n'a pas modifié significativement le comportement éthique de nos collègues. Il n'y a ainsi ni succès ni échec dans tout ce processus, mais une lente progression vers un système clarifié, contraignant à des réflexions économiques tout en respectant la primauté des impératifs cliniques. 